\title{
Article \\ Characteristics of the AT-Hook Motif Containing Nuclear Localized $(A H L)$ Genes in Carrot Provides Insight into Their Role in Plant Growth and Storage Root Development
}

\author{
Gabriela Machaj and Dariusz Grzebelus * \\ Department of Plant Biology and Biotechnology, Faculty of Biotechnology and Horticulture, \\ University of Agriculture in Krakow, 31-120 Krakow, Poland; gabriela.machaj@student.urk.edu.pl \\ * Correspondence: dariusz.grzebelus@urk.edu.pl; Tel.: +48-12-662-5191
}

Citation: Machaj, G.; Grzebelus, D. Characteristics of the AT-Hook Motif Containing Nuclear Localized (AHL) Genes in Carrot Provides Insight into Their Role in Plant Growth and Storage Root Development. Genes 2021, 12, 764. https://doi.org/ $10.3390 /$ genes 12050764

Academic Editor: Alice Hayward

Received: 29 March 2021

Accepted: 13 May 2021

Published: 18 May 2021

Publisher's Note: MDPI stays neutral with regard to jurisdictional claims in published maps and institutional affiliations.

Copyright: (c) 2021 by the authors. Licensee MDPI, Basel, Switzerland. This article is an open access article distributed under the terms and conditions of the Creative Commons Attribution (CC BY) license (https:// creativecommons.org/licenses/by/ $4.0 /)$.

\begin{abstract}
The AT-hook motif containing nuclear localized (AHL) gene family, controlling various developmental processes, is conserved in land plants. They comprise Plant and Prokaryote Conserved (PPC) domain and one or two AT-hook motifs. DcAHLc1 has been proposed as a candidate gene governing the formation of the carrot storage root. We identified and in-silico characterized carrot AHL proteins, performed phylogenetic analyses, investigated their expression profiles and constructed gene coexpression networks. We found $47 \mathrm{AHL}$ genes in carrot and grouped them into two clades, A and B, comprising 29 and 18 genes, respectively. Within Clade-A, we distinguished three subclades, one of them grouping noncanonical AHLs differing in their structure (two PPC domains) and/or cellular localization (not nucleus). Coexpression network analysis attributed AHLs expressed in carrot roots into four of the 72 clusters, some of them showing a large number of interactions. Determination of expression profiles of $A H L$ genes in various tissues and samples provided basis to hypothesize on their possible roles in the development of the carrot storage root. We identified a group of rapidly evolving noncanonical AHLs, possibly differing functionally from typical AHLs, as suggested by their expression profiles and their predicted cellular localization. We pointed at several AHLs likely involved in the development of the carrot storage root.
\end{abstract}

Keywords: AT-hook motif; coexpression network analysis; Daucus carota L; development; AHL gene family; PPC domain; phylogeny; transcriptome

\section{Introduction}

The AT-hook motif containing nuclear localized (AHL) gene family is common and conserved in all land plants, suggesting that $A H L$ genes are important for plant growth and development. However, little is known about their precise roles. Functional analysis of some AHLs has been mostly limited to Arabidopsis thaliana. Some AtAHL genes have been described as controlling hypocotyl growth and senescence [1-3], fertility and pollen development [4,5], root development [6] and flowering [7,8]. Additionally, they may be involved in the regulation of primary metabolism [9], hormonal homeostasis and signaling [10,11], drought tolerance [11] and pathogen defense [12]. Beside A. thaliana, an AHL gene was reported to be required for the development of maize ears [13]; depressed palea1 (dp1) gene involved in floral development in rice was attributed to the AHL family [14] and members of the $A H L$ gene family were reported as controlling pathogen defense response in chickpea [15].

On the structural level, AHL proteins comprise two conserved units, ca. 120-aa-long Plant and Prokaryote Conserved (PPC) domain (previously annotated as DUF296) and a region carrying one or two AT-hook motifs [3]. The AT-hook motif contains a core sequence Arg-Gly-Arg (flanked by Arg-Lys/Pro) capable of binding to AT-rich regions of B-form DNA [16]. AT-hook motifs have been divided into two types depending on the sequence variation at the C-terminus of the Arg-Gly-Arg core. Type-I contains Gly-Ser-Lys-Asn-Lys 
consensus sequence whereas Type-II carries Arg-Lys-Tyr [17]. The PPC domain is located at the carboxyl end relative to the AT-hook motif(s) [17]. The PPC domain is responsible for protein-protein interactions, facilitating the formation of trimers with other AHLs (forming homo-complexes), as well as with other nuclear proteins (e.g., histones and transcription factors), resulting in hetero-complexes. The conserved core of the PPC domain (Gly-ArgPhe-Glu-Ile-Leu) seems to be essential for complex formation with transcription factors [3]. Two types of the PPC domain are distinguished depending on the amino acid sequence of the region upstream the conserved core motif. Type-A contains conserved Leu-Arg-Ser-His sequence whereas in Type-B Phe-Thr-Pro-His is the conserved sequence. Thus, based on the composition of AT-hook motif(s) and the type of the PPC domain, AHL proteins have been classified into three subtypes. Type-I AHLs contain one AT-hook motif of Type-I and Type-A PPC domain, Type-II AHLs contain two AT-hook motifs (Type-I and Type-II) and Type-B PPC domain, whereas Type-III AHLs contain one Type-II AT-hook motif and one Type-B PPC domain. Phylogenetic analysis of AHL gene families from 19 land plant species indicated that all Type-I AHLs belonged to Clade-A while Type-II and Type-III AHLs were grouped in Clade-B [18].

The cultivated carrot (Daucus carota subsp. sativus) is among the top 10 vegetable crops globally in terms of the area of production and market value. The worldwide carrot production grows steadily, possibly owing to increased awareness of health benefits associated with carrot consumption [19]. The carrot genome, comprising $473 \mathrm{Mb}$, has been recently sequenced [20] and a member of the AHL family, named DcAHLc1, has been proposed as a candidate gene governing the formation of the fleshy storage root [21]. However, the $A H L$ gene family in carrot has not been thoroughly characterized. Here, we present global structural and functional characteristics of the $A H L$ gene family in carrot, providing insight into the role of $A H L s$ in developmental processes, including their possible involvement in the development storage roots.

\section{Materials and Methods}

\subsection{Identification and In-Silico Characterization of AHL Proteins}

Protein and transcript sequences from the carrot reference genome [20] were retrieved from NCBI (NCBI Annotation Release 100, 27 June 2016). InterProScan 5 v.5.3876.0 [22] (with options: -appl Pfam; -dp; -iprlookup) was used to mine for carrot proteins carrying the PPC/DUF296 domain (Pfam ID PF03479; Available online: https: / / pfam.xfam.org/ (accessed on 7 October 2020)). The presence of the domain was further confirmed using the Conserved Domains Database (Available online: https: / www.ncbi. nlm.nih.gov/Structure/cdd/wrpsb.cgi (accessed on 15 September 2020)). Motifs in protein sequences were predicted by MEME v.5.1.0 [23] with e-value $10^{-8}$ and parameters: -protein -oc. -nostatus -time 18,000 -mod anr -nmotifs 10 -minw 4 -maxw 160 -objfun classic -markov_order 0. Unique sequences containing both PPC domain(s) and AT-hook motif(s) were selected as representatives of the $A H L$ gene family. Protein isoelectric points $(\mathrm{pI})$ and molecular weight (MW) were predicted using Expasy Server (Available online: https:/ / web.expasy.org/compute_pi / (accessed on 16 September 2020)) whereas subcellular localization and signal peptides were predicted using CELO v.2.5 [24], (Available online: http:/ / cello.life.nctu.edu.tw / (accessed on 16 September 2020)) and SignalP 5.0 Server [25], (Available online: http:/ / www.cbs.dtu.dk/services/SignalP/ (accessed on 16 September 2020)), respectively. Structures of the carrot $A H L$ genes were determined by aligning respective coding sequences to the reference genome assembly [20] (NCBI accession LNRQ01000000).

\subsection{Phylogenetic Analysis}

To infer the evolution of carrot AHLs, we retrieved 29 A. thaliana AHL protein sequences from the TAIR database (Available online: https://www.arabidopsis.org/ (accessed on 21 September 2020) and the PPC domain of Escherichia coli from InterPro database (Available online: https://www.ebi.ac.uk/interpro/ (accessed on 21 September 
2020)), and used them together with the carrot AHLs to construct a phylogenetic tree. All protein sequences (represented by the longest isoforms) were aligned in MUSCLE [26] using default parameters. MEGA-X [27] was used to find the best model and to construct a maximum likelihood (ML) tree based on 1000 bootstrap replicates.

\subsection{Gene Duplication Events}

Carrot AHL proteins were used as queries for BLASTp against the carrot protein database at e $\leq 1 \times 10^{5}$. To identify whole genome, segmental, tandem, proximal, and dispersed gene duplication events we used the top five hits for the duplicate_gene_classifier function in MCScanX [28] using default parameters.

\subsection{Analysis of Gene Expression}

We analyzed differential expression patterns of the carrot $A H L$ genes using previously reported transcriptomic data from the wild and the cultivated carrots [29]. To determine tissue-specific vs. constitutive gene expression patterns, we also used transcriptomic data from 20 different tissues of the reference carrot line DH1 [20] (PRJNA291977). Kallisto v.0.44.0 [30] was used to estimate the expression of AHL genes in each tissue separately. Read counts were standardized using median-by-ratio normalization implemented in EBSeq v.1.12.0 package [31] and then log + 1-transformed.

\subsection{Gene Coexpression Network Analysis}

To develop coexpression networks, we used 47 transcriptomic data from the wild (9 samples) and the cultivated carrots (9 samples) [29] and 29 carrot root tissues [32] (BioProject PRJNA350691). Reads counts obtained from Kallisto v.0.44.0 [30] were normalized using the median normalization method in the EBSeq v.1.12.0 R package [31]. Correlations between carrot $A H L$ genes and other genes differentially expressed during cultivated carrot development, as well as between the cultivated and the wild carrots (FDR < 0.05) [29], were calculated in Psych v.2.0.9 R package [33] using Pearson correlation and filtered by adjusted $p$-value $<0.01$. Network statistics were calculated in Igraph v.1.2.6 R package [34]. Cytoscape v.3.7.2 [35] and clusterMaker v.1.3.1 plug-in [36] were used to construct coexpression networks of $A H L$ genes and coexpressed genes. Functional annotation of all carrot genes was made using GO FEAD [37]. Analysis and visualization of Gene Ontology terms in the constructed networks was performed in Cytoscape with ClueGO v.2.5.7 [38] plug-in.

\section{Results and Discussion}

\subsection{Identification and Characterization of Carrot AHLs}

According to the annotation of the carrot reference genome (NCBI Annotation Release 100, 27 June 2016), there were 61 AHL genes. However, only 45 of them encoded proteins carrying both the PPC-domain and the AT-hook motif(s). Using a genome-wide scan, we also identified one additional gene (LOC108192665) that also encoded a protein comprising both AHL functional units. Additionally, in subsequent analyses we also included a gene (LOC108207572) which, according to results produced by InterProScan, encoded a protein with an incomplete PPC-domain and an AT-hook motif. Thus, we further characterized 47 putative carrot $A H L$ genes (Table 1, Table S1 in the Supplementary Materials).

Carrot AHL proteins carried one or two AT-hook motifs of Type-I or Type-II and one or two PPC domains (Figure 1). The conserved amino acid core of the PPC domain (Gly-Arg-Phe-Glu-Ile-Leu) was found in ca. $40 \%$ of the analyzed proteins, as compared to $62 \%$ in A. thaliana [3]. 
Gene structure

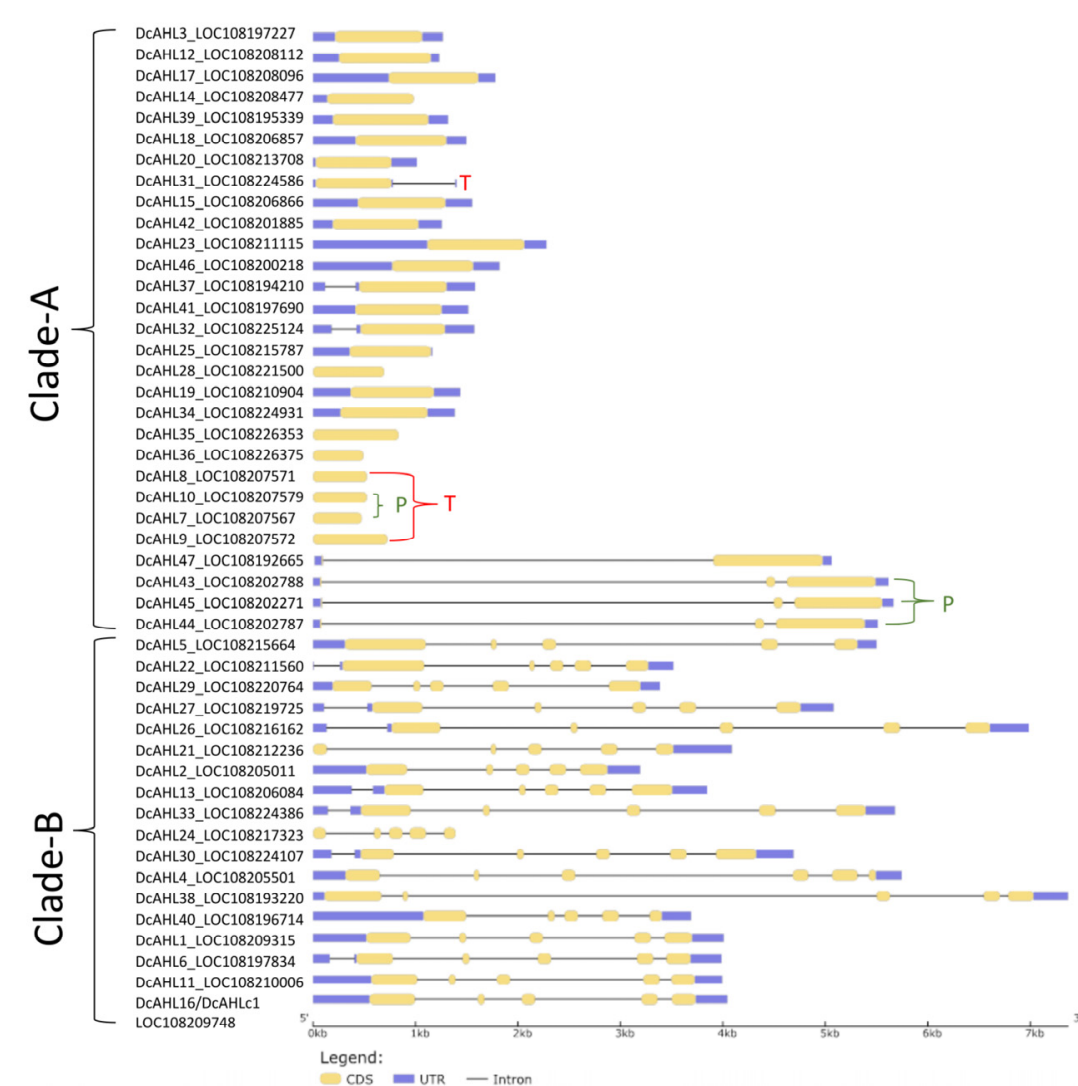

Protein structure

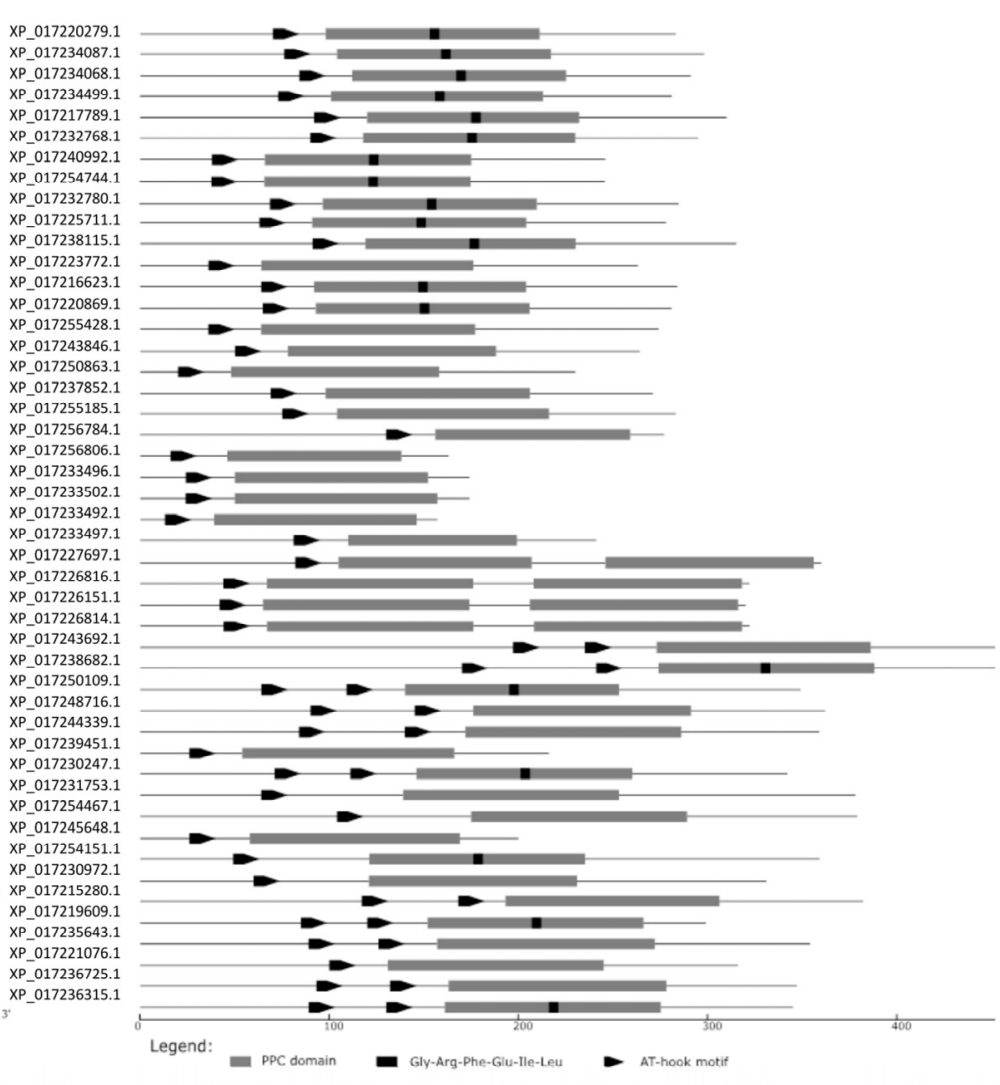

AT-hook motif PPC

Type-I Type-II domain

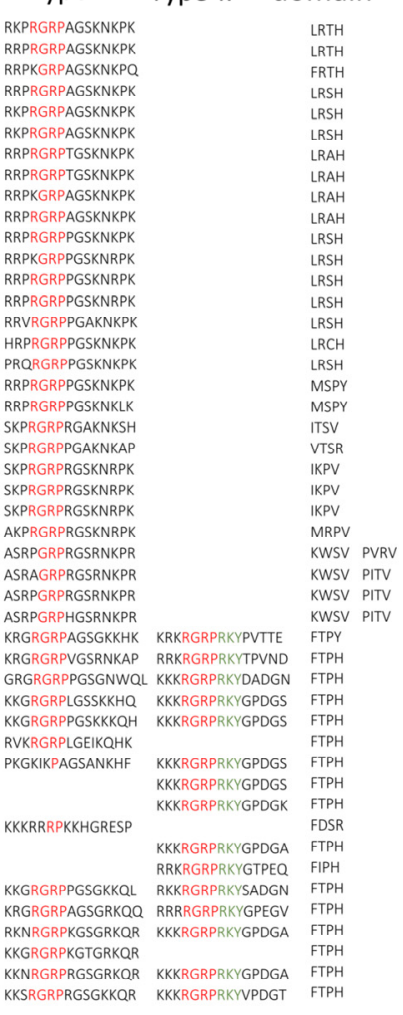

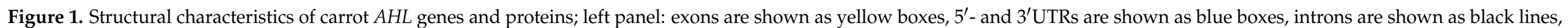

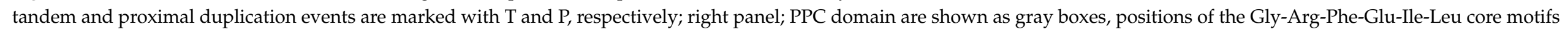

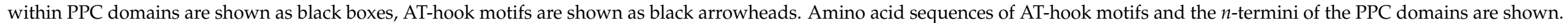


Phylogenetic analysis of AHLs from carrot and A. thaliana showed diversification of carrot AHLs into two major clades, as described previously in other species $[18,19,39]$, Clade-A and Clade-B, comprising 29 and 18 carrot AHLs, respectively (Figure 2).

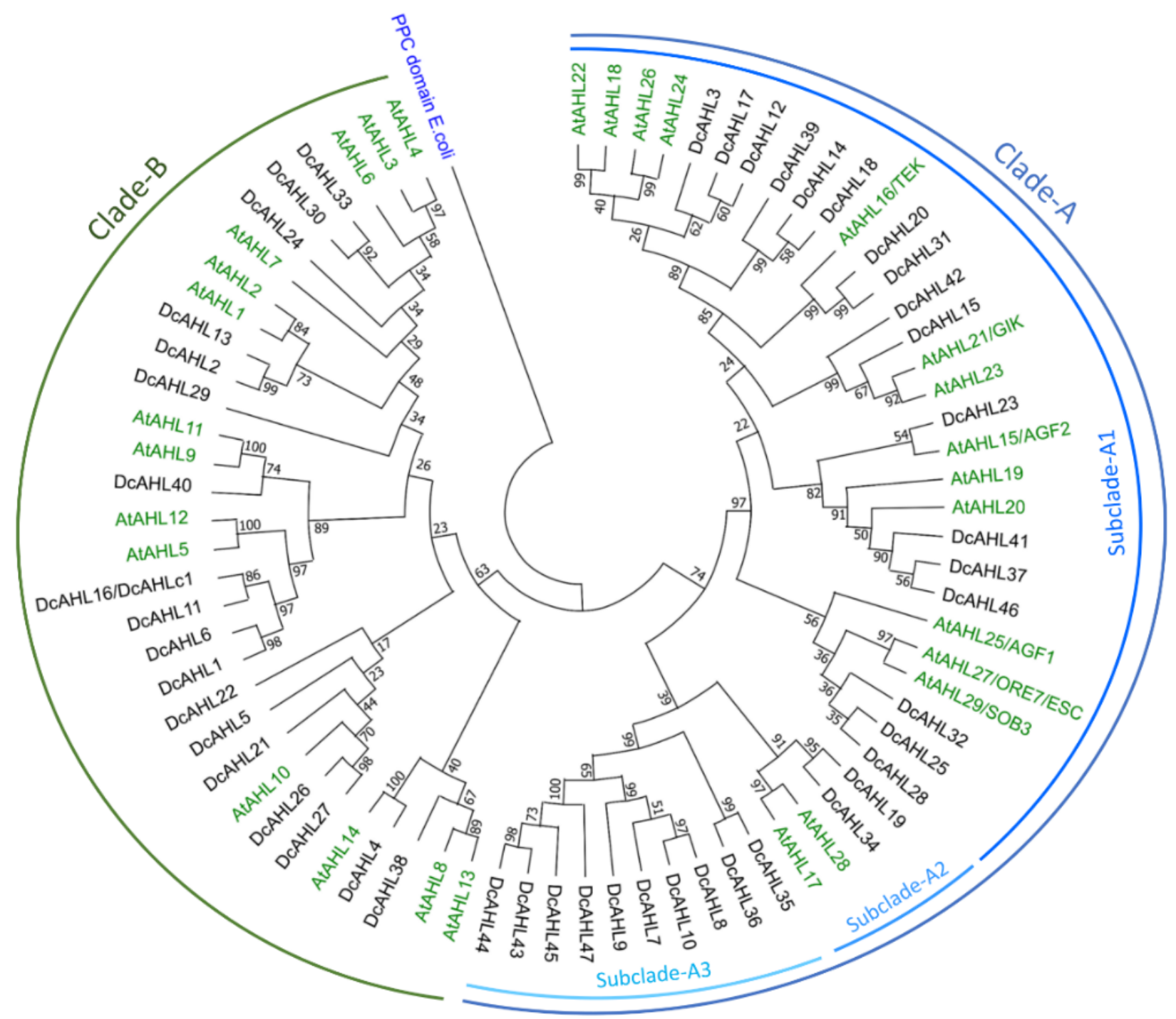

Figure 2. Maximum likelihood tree (1000 bootstrap replicates) of AHL proteins in carrot (black) and A. thaliana (green); PPC domain from E. coli (blue) was used to root the tree.

Within Clade-A three subclades, A1, A2, and A3, could be distinguished. The canonical Type-I AHLs were grouped in subclade A1, containing 17 carrot proteins showing clear relationships to their homologs from A. thaliana. All of them contained one Type-I AT-hook motif Arg-Gly-Arg-Pro, followed by Gly-Ser-Lys-Asn-Lys-Pro-Lys on the C-terminus and Type-A PPC domain starting with Leu-Arg-Ser-His on the $n$-terminus (Figure 1). The remaining 12 proteins assigned to subclades A2 and A3 had shorter and less conserved PCC domains (Figure 1) and showed different characteristics. In subclade A2, carrot DcAHL19 and DcAHL33 clustered with AtAHL17 and AtAHL28, while carrot proteins in subclade A3, carrying one AT-hook motif and one or two PPC domains, were not associated with any AHL from $A$. thaliana (Figure 2). 
Table 1. List of $A H L$ genes identified in carrot.

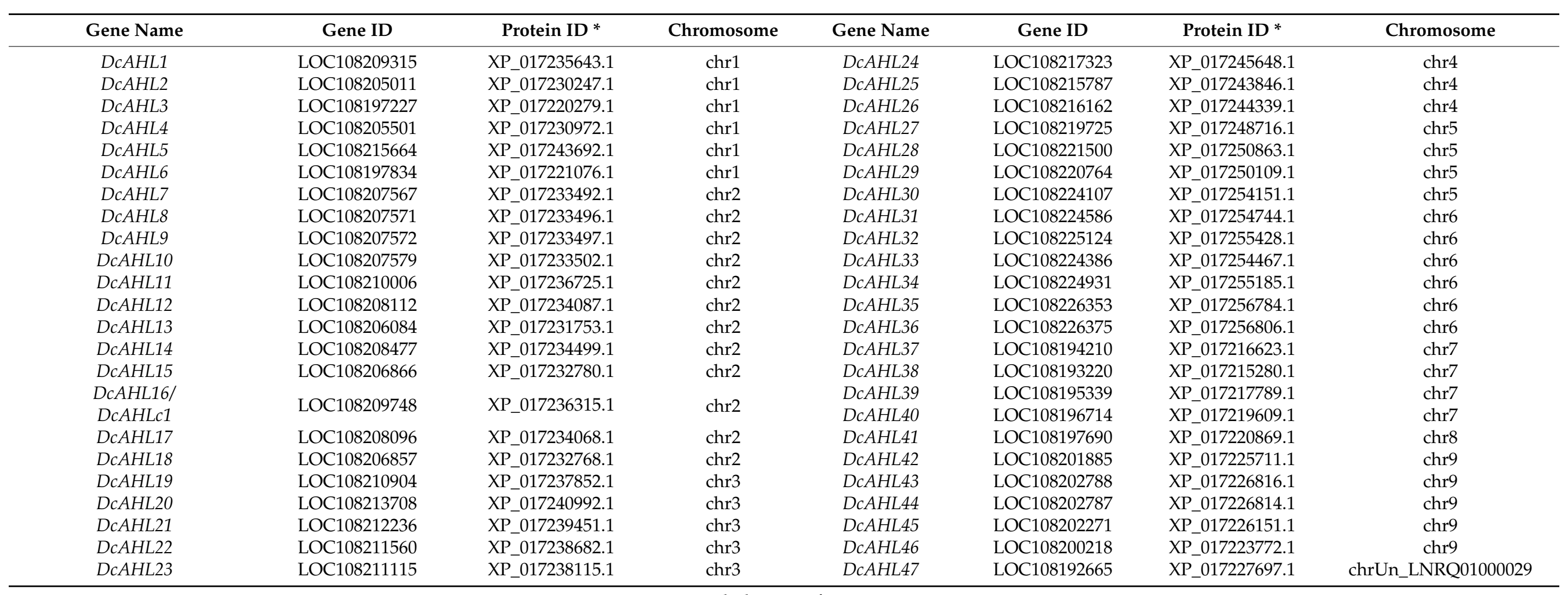


In Clade-B, AHLs carried Type-B PPC domain with a highly conserved sequence Phe-Thr-Pro-His on the $n$-terminus and one or two AT-hook motifs (Figure 1). Depending on the AT-hook motif composition, proteins assigned to Clade-B could be divided into Type-II and Type-III [3]. In Type-II, most carrot AHLs proteins (61\%) contained two types of the AT-hook motif, i.e., Type-I with a conserved core Arg-Gly-Arg-Pro followed by less conserved amino acids (Figures 1 and 3B), and Type-II with a highly conserved ArgGly-Arg-Pro-Arg-Lys-Tyr core (Figures 1 and 3C). Generally, Type-I AT-hook motifs in Clade-B were less conserved than those in Clade-A (Figure 3A,B). Three proteins carrying only one Type-I AT-hook motif also clustered with Type-II AHLs (Figure 1). A similar observation has been reported previously for two maize AHLs [40]. Type-III AHLs contained only one AT-hook motif of Type-II with a conserved Arg-Gly-Arg-ProArg-Lys-Tyr core (Figures 1 and 3B). As in other plant species [18,19,39,40], Type-II and Type-III carrot AHLs did not form distinct subclades within Clade-B (Figure 2).
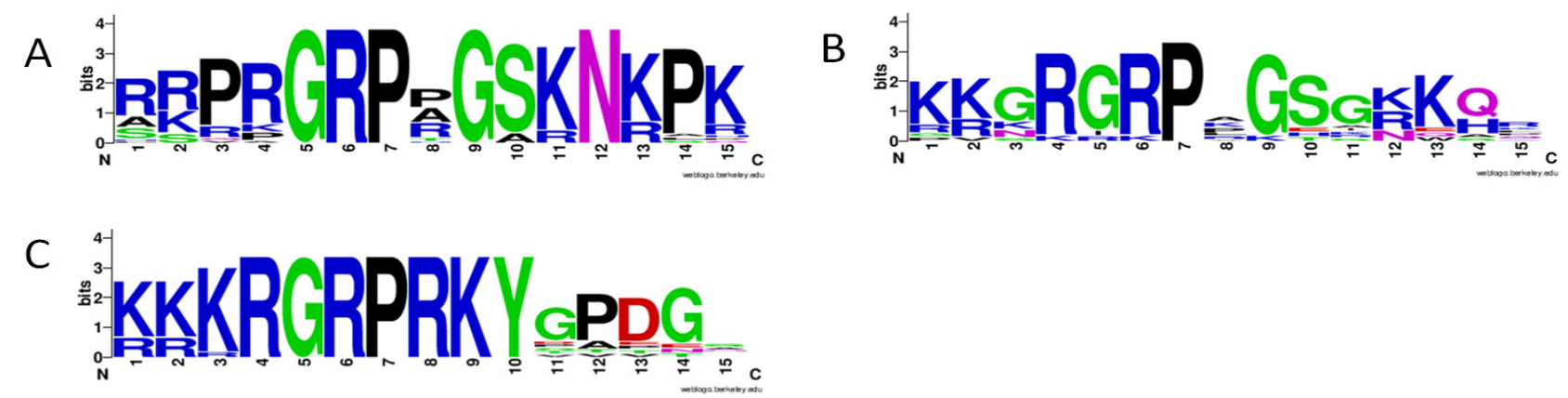

Figure 3. AT-hook motif types present in carrot AHL proteins: Type-I in Clade-A (A); Type-I in Clade-B (B); Type-II (C).

The lack of separation of Type-II/III AHLs and the presence of proteins with one AThook motif of Type-I within Type-II AHLs, jointly support relatively recent divergence of these two protein types and multiple transitions between Type-II and Type-III. Phylogeny of $A$. thaliana and carrot AHLs support the hypothesis that Clade-B evolved from CladeA $[3,22,23]$.

\subsection{Structure of AHL Genes}

As expected, genes grouped in subclades A1 and A2 (canonical Clade-A AHLs) were intronless, whereas in Clade-B Type-II AHLs contained five exons and in Type-III AHLs the number of exons ranged from five (three genes) to six (one gene) (Figure 1). Positions of exon/intron junctions in Clade-B genes were conserved. Within subclade A3, six genes, DcAHL7-DcAHL10, DcAHL35 and DcAHL36, were intronless, while the remaining four, DcAHL43, DcAHL44, DcAHL45, and DcAHL47, showed an unusual structure, as they all harbored two introns, with exceptionally large intron I, and carried two PPC domains (Figure 1).

\subsection{Duplication Events}

Gene families in plants may enlarge either as a result of whole genome duplication (WGD) events or by segmental duplication events including tandem, proximal and dispersed duplications [41]. In carrot, two lineage-specific WGDs have contributed to the expansion of carrot gene families [20]. Similar to maize, $A$. thaliana and rice [40,42], mostly dispersed duplications contributed the divergence of the $A H L$ gene family in carrot. Thirtyseven (ca. 80\%) carrot AHLs evolved through dispersed duplications. Besides dispersed duplication events, we also observed four tandem and five proximal gene duplications contributing to the $A H L$ gene expansion in carrot (Figure 1). Interestingly, most of them were observed within subclade $\mathrm{A} 3$, possibly pointing at a recent dynamic evolution of those noncanonical AHLs in carrot. Proximal duplication events were revealed for two groups, i.e., DcAHL7 and DcAHL10 located on chromosome 2, and DcAHL43, DcAHL44 
and DcAHL45 located on chromosome 9. The latter three genes sharing the unusual structure (two introns and two PPC domains) were expressed in germinating seeds (Figure 4). DcAHL10 had high or moderate expression in most tissues while its proximal copy DcAHL7 showed no expression. Possibly, expression of DcAHL7 may be triggered environmentally, e.g., by an abiotic or biotic stress, or the gene could be nonfunctional. Tandem duplication events were detected for DcAHL8 and DCAHL9, located on chromosome 2. DcAHL8 was weakly expressed in flowers and seeds, whereas its counterpart DcAHL9 exhibited moderate expression levels in most tissues (Figure 4), possibly pointing at their sub- or neo-functionalization, supported also by different predicted cellular localizations of DcAHL8 and DcAHL9 proteins, as described below.

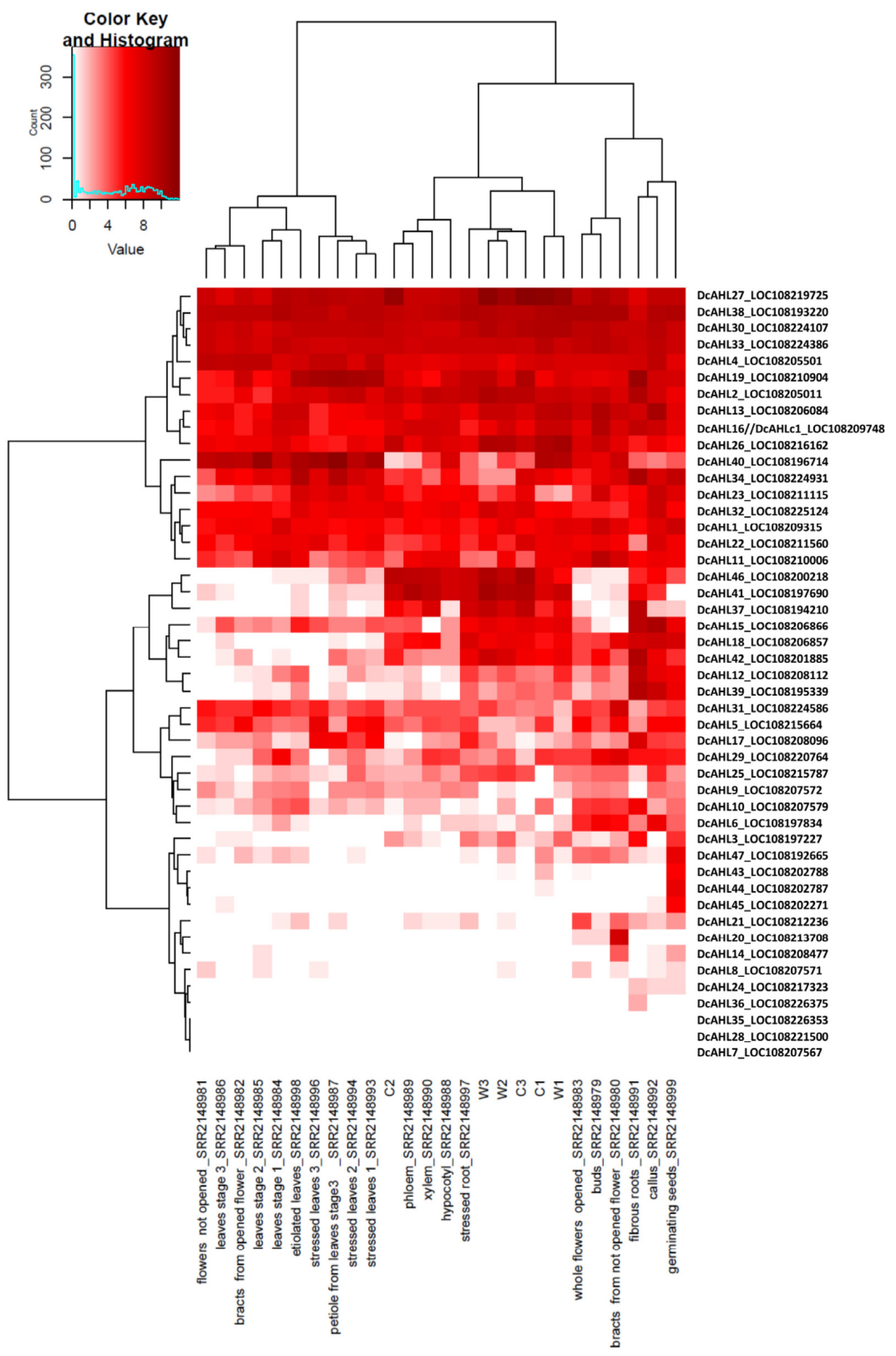

Figure 4. Hierarchical clustering and expression patterns of $A H L$ genes in seedlings (C1, W1), developing (C2, W2) and mature $(\mathrm{C} 3, \mathrm{~W} 3)$ roots from cultivated (C) and wild carrot (W) (transcriptome data from [29]) and 20 tissues of the carrot reference line DH1 (germinating seeds, hypocotyl, leaves stage 1, 2, and 3, petioles from leaves at stage 3, stressed leaves stage 1, 2, and 3, etiolated leaves, fibrous root, root xylem, root phloem, stressed root, buds, flowers not opened, whole flowers opened, bracts from not opened flowers, bracts from opened flowers, and callus; transcriptome data from [20]). The color scale represents $\log 2$ (normalized_reads +1 ) values. 


\subsection{Properties of the Predicted Proteins}

Based on in-silico predictions, carrot AHL proteins were on average 300 aa long, ranging from 157 aa (XP_017233492.1) to 454 aa (XP_017243692.1). The predicted average molecular weight of carrot AHL proteins was $31.11 \mathrm{kDa}$ with an isoelectric point (pI) between 4.41 and 10.24 (Table S2 in the Supplementary Materials). Thirty-seven DcAHLs produced proteins which were predicted to be localized in the nucleus, (consistent with their ability to bind AT-rich regions of DNA [16]). However, some proteins encoded by $D c A H L s$, mostly those grouped in subclade A3, were predicted to be targeted towards other cellular components, e.g., the plasma membrane (five proteins, including the four proteins from subclade A3 carrying two PPC domains, i.e., DcAHL43, DcAHL44, DcAHL45, and DcAHL47), chloroplasts (four proteins, including the three proteins from subclade A3, i.e., DcAHL7, DcAHL8 and DcAHL10) and extracellular space (DcAHL9 from subclade A3) (Table S2 in the Supplementary Materials). The plastid localization of the four DcAHL proteins may suggest they interact with cpDNA [40], while the predicted extracellular localization of DcAHL9, carrying a signal peptide (Sec/SPI) $(p=0.4727)$ with a cleavage site ASG-LP between positions 29 and 30 (Figure S1 in the Supplementary Materials), is surprising.

\subsection{Expression of AHL Genes}

In order to examine expression patterns of $A H L$ genes in carrot, we collated transcriptomic data from $20 \mathrm{DH}$ samples derived from various tissues and organs of carrot (data from [20]) with those obtained for wild and cultivated carrot seedlings, developing roots and mature roots [29].

Three of the 47 carrots $A H L$ genes (DcAHL7, DcAHL28, DcAHL35) were not expressed in any of the analyzed samples (Figure 4). Nineteen carrot $A H L$ genes (40\%) exhibited constitutive, high or moderate levels of expression across all examined samples (Figure 4). Fourteen of the 19 constitutively expressed AHLs belonged to Clade-B. In that clade, only four genes, i.e., DcAHL6, DcAHL21, DcAHL24, and DcAHL29, were not constitutively expressed. In contrast, in Clade-A only five genes DcAHL19, DcAHL23, $D c A H L 31, D c A H L 32$, and DcAHL34 were constitutively expressed, while the remaining genes showed tissue-specific expression.

Expression-based clustering of carrot $A H L s$ revealed two clusters, one grouping constitutively expressed AHLs and the other grouping two constitutively expressed and all tissue-specific $A H L s$ (Figure 4). Within the second cluster one can distinguish a subcluster comprising eight genes (DcAHL12, DcAHL15, DcAHL18, DcAHL41, DcAHL37, DcAHL39, DcAHL42, and DcAHL46) expressed primarily in roots. In particular, DcAHL37, DcAHL41, and DcAHL46 were highly expressed in carrot roots, while they showed little or no expression in other organs.

Hierarchical clustering based on the expression of $A H L$ genes in different organs revealed three clusters. Cluster I grouped samples from leaves, petioles, unopened flowers and bracts from open flowers. Cluster 2 grouped samples from seedlings and roots at different stages of development. In that cluster, a group of samples comprising developing cultivated roots (C2), phloem, xylem and hypocotyls formed a subcluster, while samples representing wild roots, mature storage roots and seedlings formed a separate subcluster. Cluster 3 grouped samples from opened flowers, buds, bracts from unopened flowers, fibrous roots, callus and germinating seeds.

The clustering results indicate that $A H L$ genes are developmentally regulated. Nineteen of those were expressed in all examined tissues/organs, albeit at different levels, while 25 exhibited tissue/organ-specific expression varying in the course of development. These results provide insight into possible biological roles of specific AHLs in the development of carrot organs and constitute the basis for further investigation. 


\subsection{Coexpression Networks of AHL Genes Expressed in the Storage Root}

The coexpression analysis was performed on 7794 genes identified as differentially expressed in the roots of cultivated and wild carrots, as well as during storage root development in cultivated carrots, as reported previously by Machaj et al. (2018) [29], including 35 DcAHL genes expressed in carrot roots. In the final network, 15 DcAHLs were not considered due to low correlation and high corresponding $p$-values. The coexpression analysis resulted in the construction of an extensive network containing 6798 nodes grouped into 72 clusters (Figure S2, Tables S3 and S4 in the Supplementary Materials). Correlation values in the network ranged from -0.968 to -0.766 for negatively correlated genes and from 0.766 to 1 for positively correlated genes. DcAHLs in the network were assigned to four clusters (Figure 5; yellow nodes).

Cluster-1 (Figure S2 (green) in the Supplementary Materials) comprised 3743 genes including nine AHLs (Table 2; Figure 5A) (clustering coefficient 0.519). Gene ontology (GO) analysis for Cluster-1 indicated photosynthesis, pigment biosynthetic process, nucleic acid metabolic process, cellular component/cell wall biogenesis, regulation of RNA biosynthetic process, carbohydrate metabolic process and cell division as the most enriched biological terms (Table S5 in the Supplementary Materials). DcAHLs from Cluster-1 showed 229 direct interactions (Figure 5A, Table S6 in the Supplementary Materials). While 218 interactions were within Clusters-1, 8 and 3 interactions with genes from Cluster-2 and Cluster-13 were observed, respectively. DcAHL16 was coexpressed with 62 genes. GO analysis of genes coexpressed with DcAHL16 indicated its involvement in fucose/hexose, amino sugar and nucleotide sugar metabolism; steroid biosynthesis and protein import to nucleus (Figure 6A). The high number of connections for DcAHL16 indicated that it was a prominent regulatory gene in Cluster-1. Interestingly, this gene (reported as DcAHLc1 [21]) was previously identified as a candidate domestication gene and shown to be differentially expressed during root development in cultivated and wild carrots [29]. DcAHL11, interacting with 51 genes, is another important gene in Cluster-1. GO analysis of the first neighbors of DcAHL11 implied that the gene was likely involved in the regulation of cell division, cellulose metabolism and protein phosphorylation (Figure 6B).

Table 2. A list of $A H L$ genes showing significant interactions in the coexpression network analysis of genes differentially expressed in the carrot storage roots.

\begin{tabular}{|c|c|c|c|c|c|}
\hline Gene Name & GeneID & Cluster & Betweenness & Degree & Rank_stat \\
\hline DcAHL16//DcAHLc1 & LOC108209748 & 1 & 632.31 & 62 & 2695.25 \\
\hline DcAHL11 & LOC108210006 & 1 & 757.12 & 51 & 2626.50 \\
\hline DcAHL40 & LOC108196714 & 1 & 3490.89 & 46 & 3168.00 \\
\hline DcAHL46 & LOC108200218 & 1 & 1361.78 & 41 & 2700.25 \\
\hline DcAHL32 & LOC108225124 & 1 & 5.90 & 12 & 1268.00 \\
\hline DcAHL2 & LOC108205011 & 1 & 1.70 & 6 & 951.75 \\
\hline DcAHL29 & LOC108220764 & 1 & 9.82 & 6 & 1076.25 \\
\hline DcAHL1 & LOC108209315 & 1 & 0.66 & 4 & 788.75 \\
\hline DcAHL10 & LOC108207579 & 1 & 0.00 & 1 & 237.00 \\
\hline DcAHL38 & LOC108193220 & 2 & $16,399.50$ & 825 & 6140.50 \\
\hline DcAHL26 & LOC108216162 & 2 & 6491.57 & 566 & 5180.00 \\
\hline DcAHL13 & LOC108206084 & 2 & $10,024.21$ & 372 & 5299.75 \\
\hline DcAHL27 & LOC108219725 & 2 & $63,411.36$ & 271 & 5842.75 \\
\hline DcAHL30 & LOC108224107 & 2 & 6394.09 & 263 & 4611.25 \\
\hline DcAHL33 & LOC108224386 & 2 & 22.44 & 81 & 2234.00 \\
\hline DcAHL34 & LOC108224931 & 2 & 961.10 & 31 & 2474.00 \\
\hline DcAHL25 & LOC108215787 & 2 & 48.34 & 7 & 1299.25 \\
\hline DcAHL19 & LOC108210904 & 3 & 253.15 & 17 & 1894.25 \\
\hline DcAHL42 & LOC108201885 & 3 & 0.00 & 1 & 237.00 \\
\hline DcAHL37 & LOC108194210 & 4 & $14,455.31$ & 12 & 3685.00 \\
\hline
\end{tabular}

Cluster-2 (Figure S2 (red) in the Supplementary Materials) comprised 2314 genes including eight AHLs (clustering coefficient 0.615; Table 2; Figure 5B). AHLs from Cluster-2 
demonstrated 2416 direct interactions with other genes, including 2312 interactions within Cluster-2 followed by 79, 17, and 3 interactions with genes from Cluster-1, Cluster-3 and Cluster-13, respectively (Table S6 in the Supplementary Materials). GO enrichment analysis for Cluster-2 indicated that the most significantly enriched biological processes were associated with transport (ion, nitrogen compound, protein, endosomal and vesicle-mediated transport), plastid organization and tRNA/mRNA/rRNA metabolic process. A group of terms associated with root development, multicellular organism development, plant organ morphogenesis, mitotic cell cycle, cell/organ differentiation/morphogenesis and development; cell wall biogenesis and starch/polysaccharide metabolism could also be distinguished (Table S7 in the Supplementary Materials). DcAHL38 showed 825 direct interactions in Cluster-2. GO enrichment analysis indicated that it might be involved in a range of biological processes, e.g., intracellular protein transport; regulation of immune system and meristem structural organization, as well as anatomical structure morphogenesis and calcium-mediated signaling (Figure 6C). Interestingly, DcAHL38 showed the strongest correlation $\left(0.934 ; p=3.431 \times 10^{14}\right)$ with BYPASS1 (LOC108196316). In A. thaliana, $B Y P A S S 1$ is required for normal root and shoot development by preventing constitutive production of a root mobile carotenoid-derived signaling compound that is capable of arresting shoot and leaf development $[43,44]$. Moreover, DcAHL38 was coexpressed with genes LOC108214645 $\left(0.924, p=6.714 \times 10^{13}\right)$ and LOC108218757 which in A. thaliana are involved in the regulation of root stem cell niche identity [45] and responsible for normal plant growth and development [46]. Thus, DcAHL38 is possibly involved in the regulation of storage root morphogenesis in carrot. Additionally, DcAHL38 shows a positive correlation with four other $A H L$ genes (DcAHL13, DcAHL26, DcAHL27, and DcAHL30) from Cluster-2, forming a group of five interrelated DcAHLs (Figure 5F). As previously reported, AHLs regulate plant growth and development by forming homo- or hetero-trimeric complexes via their PPC domains [3]. Our results suggest that the potential direct interactions among the five DcAHL proteins might be important for the regulation of storage root development, however, experimental validation of those interactions is required to support the hypothesis.

Cluster-3 (Figure S2 (blue) in the Supplementary Materials) comprised 289 genes including two AHLs (clustering coefficient 0.253; Table 2; Figure 5C). In that cluster, DcAHL19 interacted with 17 genes (four from Cluster-3, six from Cluster-1 and seven from Cluster-2), while DcAHL42 interacted (negative correlation) with just one gene. Notably, the expression of DcAHL19 was reported as highly varying in the course of the storage root development [29]. GO analysis of Cluster-3 indicated that it was primarily associated with RNA splicing, cellular response to oxidative stress, fruit/seed development and xyloglucan metabolic process (Table S8 in the Supplementary Materials).

Cluster-4 (Figure S2 (pink) in the Supplementary Materials) comprised nine genes including DcAHL37 (clustering coefficient 0.504; Table 2; Figure 5D). In that cluster, all genes were positively correlated. DcAHL37 interacted with 12 genes, only five of them being assigned to Cluster-4. Due to the small number of genes in that cluster, it was not possible to perform GO enrichment. Nevertheless, DcAHL37 had very high 'betweenness centrality' (b.c. $=14,455.308$ ) and was highly expressed in roots (as compared to other carrot organs), which indicates that it might be important in process of the carrot storage root development. 


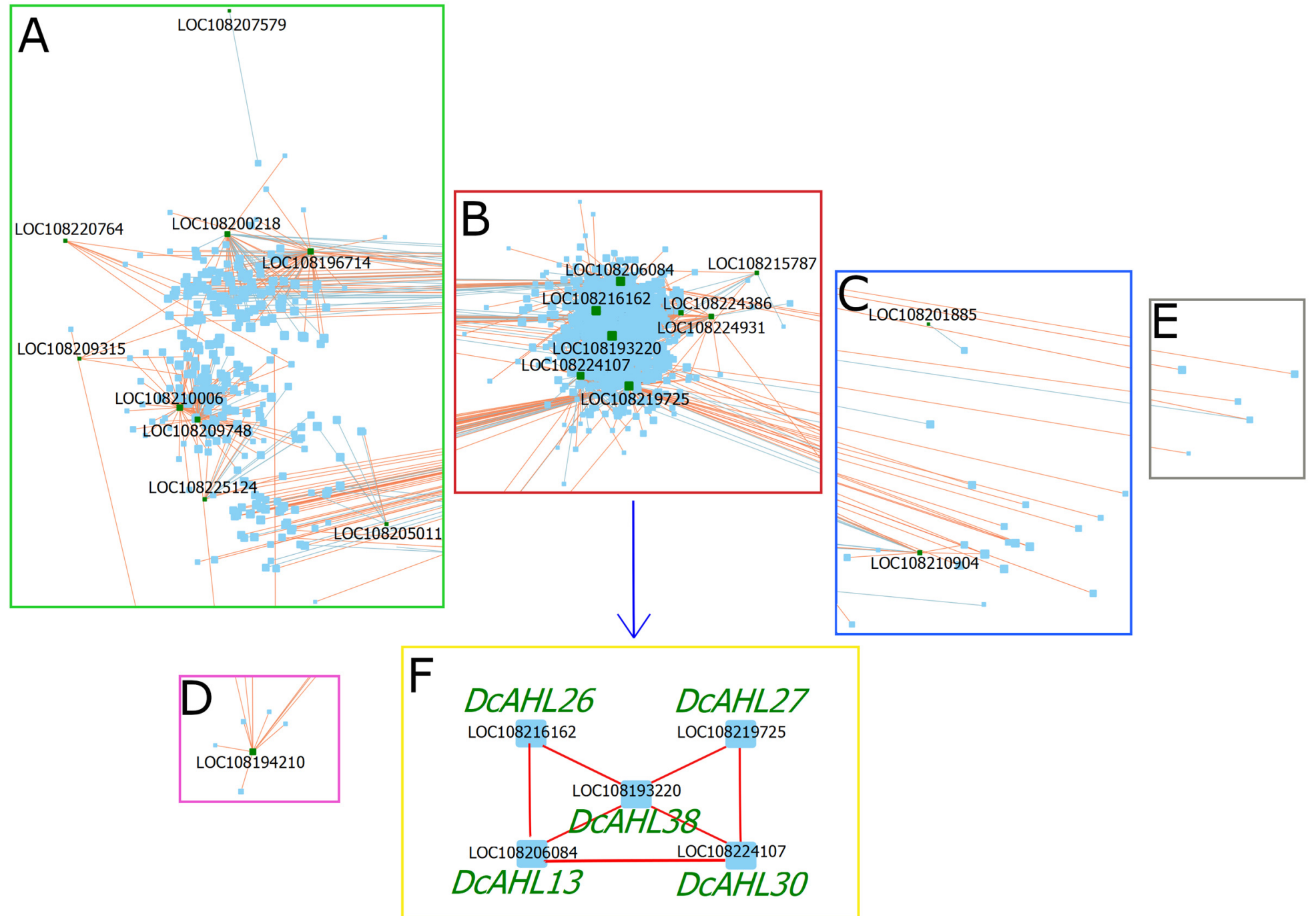

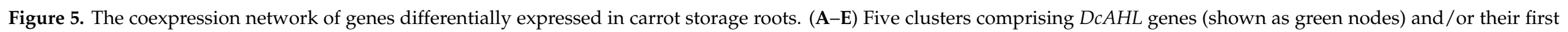

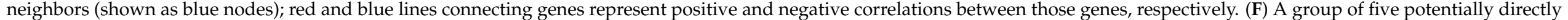
interacting DcAHL genes in Cluster-2. 

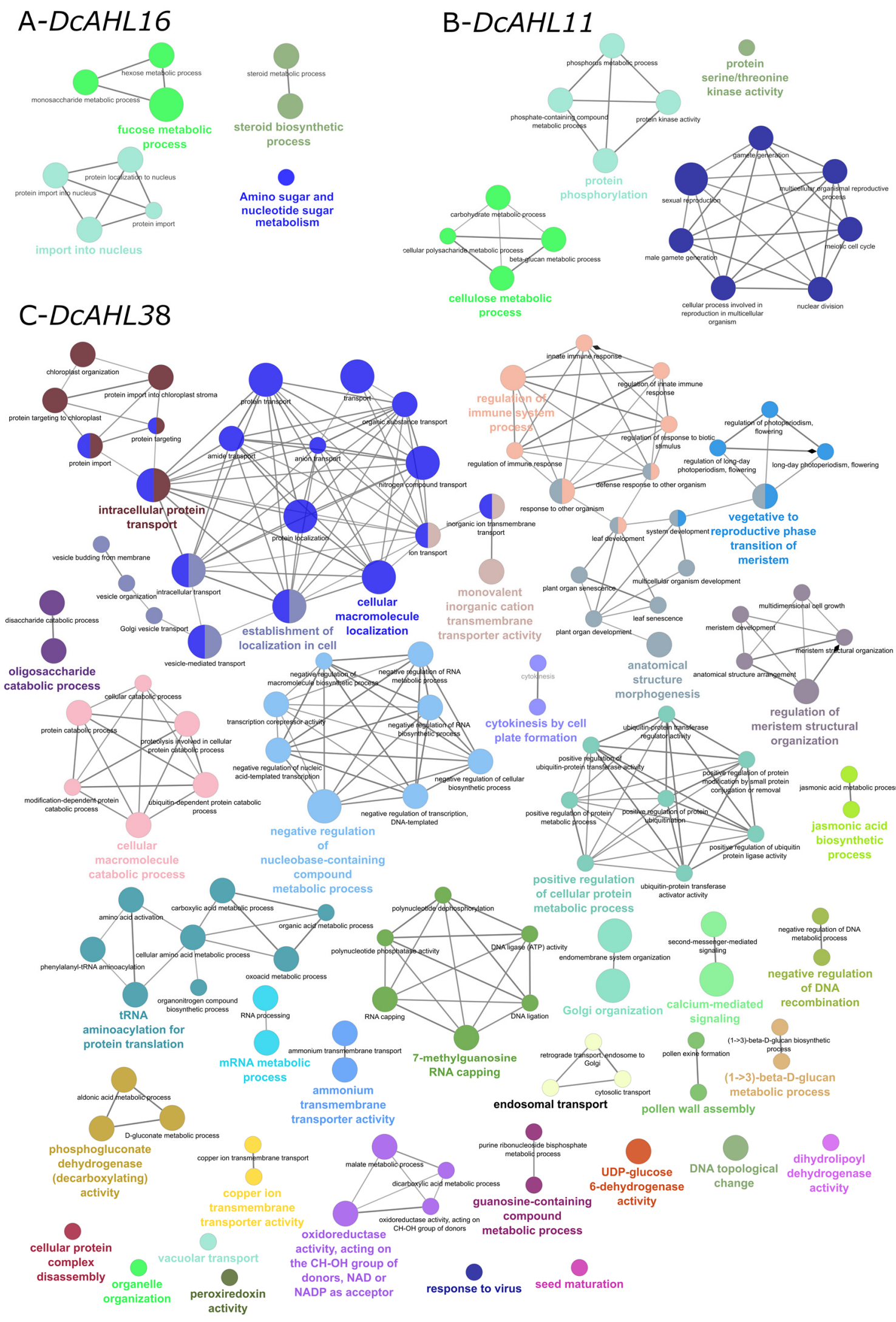

Figure 6. GO enrichment results for carrot genes possibly interacting with DcAHL16 (A); DcAHL11 (B); DcAHL38 (C). Sizes of circles show the number of genes corresponding to GO terms. 
We conclude that $D c A H L$ genes assigned to the above described clusters are likely candidates governing storage root development in carrot. To more precisely investigate their role, we performed GO enrichment analysis of all first neighbors to DcAHLs (direct interactions) and compared it with GO terms for genes assigned to clusters 5-72. For genes not coexpressed with DcAHLs, biological processes associated with macromolecule metabolism, reactive oxygen species biosynthesis, oxidation-reduction, nitrogen metabolism, methylation and post-transcriptional gene silencing were highly enriched (Figure S3 in the Supplementary Materials). Genes coexpressed with DcAHLs were mainly associated with cellular macromolecule localization, small molecule metabolism, anatomical structure development/arrangement, cell wall organization or biogenesis, biosynthesis, pigment metabolism, cell communication and division (Figure 7), suggesting that they are important determinants of the storage root development in cultivated carrots.

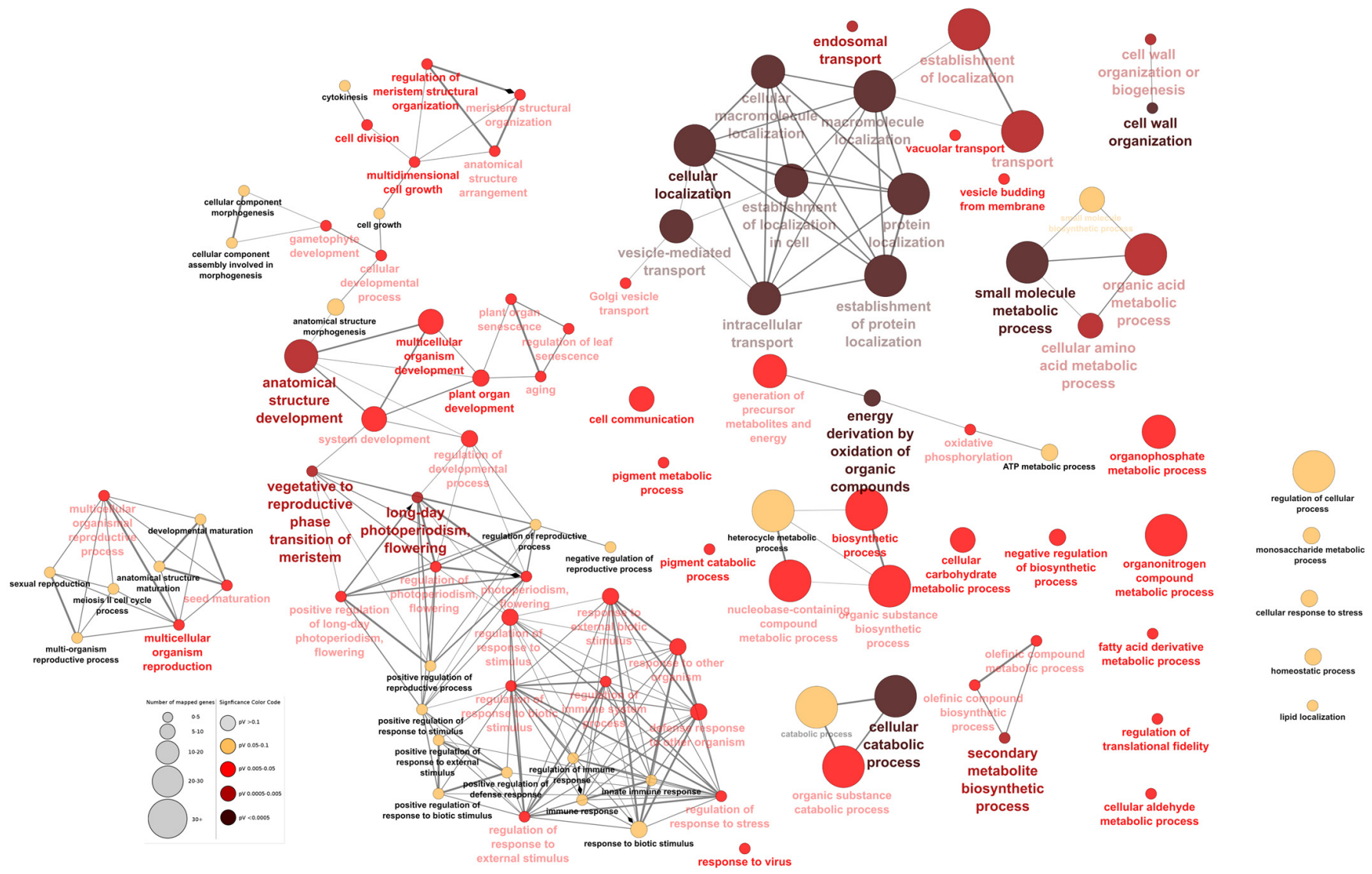

Figure 7. GO enrichment results for carrot genes possibly interacting with DcAHLs. Sizes of circles show the number of genes corresponding to GO terms, colors show significance of the enrichment, as shown in the significance legend box.

\section{Conclusions}

Using a genome-wide scan and transcriptomic evidence, we identified and characterized the $A H L$ gene family in carrot. We identified a group of noncanonical AHLs (subclade A3) which likely evolved rapidly and might have acquired functions different from typical $A H L s$, as suggested by their expression profiles and their predicted cellular localization. Gene coexpression network analysis provided evidence for the involvement of several AHLs in the development of the storage root in carrot.

Supplementary Materials: The following are available online at https:/ / www.mdpi.com/article/10.3 390/genes12050764/s1, Figure S1: A graph showing the presence of a signal peptide in XP_017233497.1 indicating a possible extracellular localization of DcAHL9, Figure S2: A graph showing a complete coexpression network of genes differentially expressed in carrot storage roots, Figure S3: ClueGO 
results for biological processes showing GO enrichment results for carrot genes in clusters not containing any DcAHL genes (clusters 5-72), Table S1: Characteristic of AHL genes in carrot, Table S2: Physical properties and cellular localization of AHL proteins, Table S3: Coexpression network—nodes, Table S4: Coexpression network-correlation, Table S5: ClueGO results for biological processes in Cluster-1, Table S6: Coexpression network-AHLs direct interactions, Table S7: ClueGO results for biological processes in Cluster-2, Table S8: ClueGO results for biological processes in Cluster-3.

Author Contributions: Conceptualization, G.M. and D.G.; methodology, G.M. and D.G.; software, G.M.; formal analysis, D.G.; investigation, G.M.; resources, G.M.; data curation, G.M.; writingoriginal draft preparation, G.M. and. D.G.; visualization, G.M.; supervision, D.G.; project administration, D.G.; funding acquisition, D.G. All authors have read and agreed to the published version of the manuscript.

Funding: Funding for research at the University of Agriculture in Krakow, Poland, was provided by the Polish Ministry of Education and Science.

Institutional Review Board Statement: Not applicable.

Informed Consent Statement: Not applicable.

Data Availability Statement: Publicly available datasets were analyzed in this study. Source files can be downloaded from the GenBank Short Read Archive acc. no. SRP155333, PRJNA291977 and PRJNA350691.

Conflicts of Interest: The authors declare no conflict of interest.

\section{References}

1. Lim, P.O.; Kim, Y.; Breeze, E.; Koo, J.C.; Woo, H.R.; Ryu, J.S.; Park, D.H.; Beynon, J.; Tabrett, A.; Buchanan-Wollaston, V.; et al. Overexpression of a chromatin architecture-controlling AT-hook protein extends leaf longevity and increases the post-harvest storage life of plants. Plant J. 2007, 52, 1140-1153. [CrossRef]

2. Street, I.H.; Shah, P.K.; Smith, A.M.; Avery, N.; Neff, M.M. The AT-hook-containing proteins SOB3/AHL29 and ESC/AHL27 are negative modulators of hypocotyl growth in Arabidopsis. Plant J. 2007, 54, 1-14. [CrossRef] [PubMed]

3. Zhao, J.; Favero, D.S.; Peng, H.; Neff, M.M. Arabidopsis thaliana AHL family modulates hypocotyl growth redundantly by interacting with each other via the PPC/DUF296 domain. Proc. Natl. Acad. Sci. USA 2013, 110, E4688-E4697. [CrossRef]

4. Lou, Y.; Xu, X.-F.; Zhu, J.; Gu, J.-N.; Blackmore, S.; Yang, Z.-N. The tapetal AHL family protein TEK determines nexine formation in the pollen wall. Nat. Commun. 2014, 5, 3855. [CrossRef] [PubMed]

5. Jia, Q.-S.; Zhu, J.; Xu, X.-F.; Lou, Y.; Zhang, Z.-L.; Zhang, Z.-P.; Yang, Z.-N. Arabidopsis AT-hook Protein TEK Positively Regulates the Expression of Arabinogalactan Proteins for Nexine Formation. Mol. Plant 2015, 8, 251-260. [CrossRef]

6. Zhou, J.; Wang, X.; Lee, J.-Y.; Lee, J.-Y. Cell-to-Cell Movement of Two Interacting AT-Hook Factors in Arabidopsis Root Vascular Tissue Patterning. Plant Cell 2013, 25, 187-201. [CrossRef] [PubMed]

7. Xu, Y.; Gan, E.-S.; Ito, T. The AT-hook/PPC domain protein TEK negatively regulates floral repressors including MAF4 and MAF5. Plant Signal. Behav. 2013, 8, e25006. [CrossRef]

8. Yun, J.; Kim, Y.-S.; Jung, J.-H.; Seo, P.J.; Park, C.-M. The AT-hook Motif-containing Protein AHL22 Regulates Flowering Initiation by Modifying FLOWERING LOCUS T Chromatin in Arabidopsis. J. Biol. Chem. 2012, 287, 15307-15316. [CrossRef]

9. Li, B.; Kliebenstein, D.J. The AT-hook motif-encoding gene METABOLIC NETWORK MODULATOR 1 underlies natural variation in Arabidopsis primary metabolism. Front. Plant Sci. 2014, 5, 415. [CrossRef]

10. Matsushita, A.; Furumoto, T.; Ishida, S.; Takahashi, Y. AGF1, an AT-Hook Protein, Is Necessary for the Negative Feedback of AtGA3ox1 Encoding GA 3-Oxidase. Plant Physiol. 2007, 143, 1152-1162. [CrossRef]

11. Wong, M.M.; Bhaskara, G.B.; Wen, T.-N.; Lin, W.-D.; Nguyen, T.T.; Chong, G.L.; Verslues, P.E. Phosphoproteomics ofArabidopsisHighly ABA-Induced1 identifies AT-Hook-Like10 phosphorylation required for stress growth regulation. Proc. Natl. Acad. Sci. USA 2019, 116, 2354-2363. [CrossRef]

12. Lu, H.; Zou, Y.; Feng, N. Overexpression of AHL20 Negatively Regulates Defenses in Arabidopsis. J. Integr. Plant Biol. 2010, 52, 801-808. [CrossRef] [PubMed]

13. Gallavotti, A.; Malcomber, S.; Gaines, C.; Stanfield, S.; Whipple, C.; Kellogg, E.; Schmidt, R.J. BARREN STALK FASTIGIATE1 Is an AT-Hook Protein Required for the Formation of Maize Ears. Plant Cell 2011, 23, 1756-1771. [CrossRef] [PubMed]

14. Jin, Y.; Luo, Q.; Tong, H.; Wang, A.; Cheng, Z.; Tang, J.; Li, D.; Zhao, X.; Li, X.; Wan, J.; et al. An AT-hook gene is required for palea formation and floral organ number control in rice. Dev. Biol. 2011, 359, 277-288. [CrossRef] [PubMed]

15. Kumar, K.; Purayannur, S.; Kaladhar, V.C.; Parida, S.K.; Verma, P.K. mQTL-seq and classical mapping implicates the role of an AT-HOOK MOTIF CONTAINING NUCLEAR LOCALIZED (AHL) family gene in Ascochyta blight resistance of chickpea. Plant Cell Environ. 2018, 41, 2128-2140. [CrossRef] [PubMed]

16. Huth, J.R.; Bewley, C.A.; Nissen, M.S.; Evans, J.N.; Reeves, R.; Gronenborn, A.M.; Clore, G.M. The solution structure of an HMG-I(Y)-DNA complex defines a new architectural minor groove binding motif. Nat. Genet. 1997, 4, 657-665. [CrossRef] 
17. Fujimoto, S.; Matsunaga, S.; Yonemura, M.; Uchiyama, S.; Azuma, T.; Fukui, K. Identification of a novel plant MAR DNA binding protein localized on chromosomal surfaces. Plant Mol. Biol. 2004, 56, 225-239. [CrossRef]

18. Zhao, J.; Favero, D.S.; Qiu, J.; Roalson, E.H.; Neff, M.M. Insights into the evolution and diversification of the AT-hook Motif Nuclear Localized gene family in land plants. BMC Plant Biol. 2014, 14, 266. [CrossRef]

19. Arscott, S.A.; Tanumihardjo, S.A. Carrots of Many Colors Provide Basic Nutrition and Bioavailable Phytochemicals Acting as a Functional Food. Compr. Rev. Food Sci. Food Saf. 2010, 9, 223-239. [CrossRef]

20. Iorizzo, M.; Ellison, S.; Senalik, D.; Zeng, P.; Satapoomin, P.; Huang, J.; Bowman, M.; Iovene, M.; Sanseverino, W.; Cavagnaro, P.; et al. A high-quality carrot genome assembly provides new insights into carotenoid accumulation and asterid genome evolution. Nat. Genet. 2016, 48, 657-666. [CrossRef]

21. Macko-Podgórni, A.; Machaj, G.; Stelmach, K.; Senalik, U.; Grzebelus, E.; Iorizzo, M.; Simon, P.W.; Grzebelus, D. Characterization of a Genomic Region under Selection in Cultivated Carrot (Daucus carota subsp. sativus) Reveals a Candidate Domestication Gene. Front. Plant Sci. 2017, 8, 415. [CrossRef] [PubMed]

22. Jones, P.; Binns, D.; Chang, H.-Y.; Fraser, M.; Li, W.; McAnulla, C.; McWilliam, H.; Maslen, J.; Mitchell, A.; Nuka, G.; et al. InterProScan 5: Genome-scale protein function classification. Bioinformatics 2014, 30, 1236-1240. [CrossRef]

23. Bailey, T.L.; Boden, M.; Buske, F.A.; Frith, M.; Grant, C.E.; Clementi, L.; Ren, J.; Li, W.W.; Noble, W.S. MEME SUITE: Tools for motif discovery and searching. Nucleic Acids Res. 2009, 37, w202-w208. [CrossRef]

24. Yu, C.-S.; Chen, Y.-C.; Lu, C.-H.; Hwang, J.-K. Prediction of protein subcellular localization. Proteins Struct. Funct. Bioinform. 2006, 64, 643-651. [CrossRef]

25. Armenteros, J.J.A.; Tsirigos, K.D.; Sønderby, C.K.; Petersen, T.N.; Winther, O.; Brunak, S.; Von Heijne, G.; Nielsen, H. SignalP 5.0 improves signal peptide predictions using deep neural networks. Nat. Biotechnol. 2019, 37, 420-423. [CrossRef]

26. Edgar, R.C. MUSCLE: Multiple sequence alignment with high accuracy and high throughput. Nucleic Acids Res. 2004, 32, 1792-1797. [CrossRef]

27. Kumar, S.; Stecher, G.; Li, M.; Knyaz, C.; Tamura, K. MEGA X: Molecular evolutionary genetics analysis across computing platforms. Mol. Biol. Evol. 2018, 35, 1547-1549. [CrossRef] [PubMed]

28. Wang, Y.; Tang, H.; DeBarry, J.D.; Tan, X.; Li, J.; Wang, X.; Lee, T.-H.; Jin, H.; Marler, B.; Guo, H.; et al. MCScanX: A toolkit for detection and evolutionary analysis of gene synteny and collinearity. Nucleic Acids Res. 2012, 40, e49. [CrossRef]

29. Machaj, G.; Bostan, H.; Macko-Podgórni, A.; Iorizzo, M.; Grzebelus, D. Comparative Transcriptomics of Root Development in Wild and Cultivated Carrots. Genes 2018, 9, 431. [CrossRef]

30. Bray, N.L.; Pimentel, H.; Melsted, P.; Pachter, L. Near-optimal probabilistic RNA-seq quantification. Nat. Biotechnol. 2016, 34, 525-527. [CrossRef]

31. Leng, N.; Dawson, J.A.; Thomson, J.A.; Ruotti, V.; Rissman, A.I.; Smits, B.M.G.; Haag, J.D.; Gould, M.N.; Stewart, R.M.; Kendziorski, C. EBSeq: An empirical Bayes hierarchical model for inference in RNA-seq experiments. Bioinformatics 2013. [CrossRef]

32. Ellison, S.; Senalik, D.; Bostan, H.; Iorizzo, M.; Simon, P. Fine Mapping, Transcriptome Analysis, and Marker Development for $\mathrm{Y}_{2}$, the Gene That Conditions $\beta$-Carotene Accumulation in Carrot (Daucus carota L.). G3 Genes Genomes Genet. 2017, 7, $2665-2675$. [CrossRef]

33. Revelle, W.R. Psych: Procedures for Personality and Psychological Research; Northwestern University: Evanston, IL, USA, 2017; R package version 2.0.9. Available online: https:/ /CRAN.R-project.org/package=psych (accessed on 20 October 2020).

34. Csardi, G.; Nepusz, T. The igraph software package for complex network research. Int. J. Complex Syst. 2006, 1695, 1-9.

35. Shannon, P.; Markiel, A.; Ozier, O.; Baliga, N.S.; Wang, J.T.; Ramage, D.; Amin, N.; Schwikowski, B.; Ideker, T. Cytoscape: A Software Environment for Integrated Models of Biomolecular Interaction Networks. Genome Res. 2003, 13, 2498-2504. [CrossRef]

36. Morris, J.H.; Apeltsin, L.; Newman, A.M.; Baumbach, J.; Wittkop, T.; Su, G.; Bader, G.D.; Ferrin, T.E. clusterMaker: A multialgorithm clustering plugin for Cytoscape. BMC Bioinform. 2011, 12, 436. [CrossRef]

37. Araujo, F.A.; Barh, D.; Silva, A.; Guimarães, L.; Ramos, R.T.J. GO FEAT: A rapid web-based functional annotation tool for genomic and transcriptomic data. Sci. Rep. 2018, 8, 1794. [CrossRef]

38. Bindea, G.; Mlecnik, B.; Hackl, H.; Charoentong, P.; Tosolini, M.; Kirilovsky, A.; Fridman, W.-H.; Pagès, F.; Trajanoski, Z.; Galon, J. ClueGO: A Cytoscape plug-in to decipher functionally grouped gene ontology and pathway annotation networks. Bioinformatics 2009, 25, 1091-1093. [CrossRef]

39. Kim, H.B.; Oh, C.J.; Park, Y.C.; Lee, Y.; Choe, S.-H.; An, C.S.; Choi, S.-B. Comprehensive analysis of AHL homologous genes encoding AT-hook motif nuclear localized protein in rice. BMB Rep. 2011, 44, 680-685. [CrossRef]

40. Bishop, E.H.; Kumar, R.; Luo, F.; Saski, C.; Sekhon, R.S. Genome-wide identification, expression profiling, and network analysis of AT-hook gene family in maize. Genomics 2020, 112, 1233-1244. [CrossRef]

41. Lawton-Rauh, A. Evolutionary dynamics of duplicated genes in plants. Mol. Phylogenetics Evol. 2003, 29, 396-409. [CrossRef]

42. Wang, Y.; Wang, X.; Tang, H.; Tan, X.; Ficklin, S.P.; Feltus, F.A.; Paterson, A.H. Modes of Gene Duplication Contribute Differently to Genetic Novelty and Redundancy, but Show Parallels across Divergent Angiosperms. PLoS ONE 2011, 6, e28150. [CrossRef] [PubMed]

43. Van Norman, J.M.; Frederick, R.L.; Sieburth, L.E. BYPASS1 Negatively Regulates a Root-Derived Signal that Controls Plant Architecture. Curr. Biol. 2004, 14, 1739-1746. [CrossRef] [PubMed] 
44. Van Norman, J.M.; Sieburth, L.E. Dissecting the biosynthetic pathway for the bypass1 root-derived signal. Plant J. 2007, 49, 619-628. [CrossRef] [PubMed]

45. Yu, Q.; Liu, J.; Zheng, H.; Jia, Y.; Tian, H.; Ding, Z. Topoisomerase II-associated protein PAT1H1 is involved in the root stem cell niche maintenance in Arabidopsis thaliana. Plant. Cell Rep. 2016, 35, 1297-1307. [CrossRef] [PubMed]

46. Bundock, P.; Hooykaas, P. An Arabidopsis hAT-like transposase is essential for plant development. Nat. Cell Biol. 2005, 436, 282-284. [CrossRef] 